\title{
Slow drag in wet-snow avalanche flow
}

\author{
B. SOVILLA, ${ }^{1}$ M. KERN, ${ }^{2}$ M. SCHAER ${ }^{3}$ \\ ${ }^{1}$ Avalanches, Debris Flows and Rockfall Research Unit, WSL Institute for Snow and Avalanche Research SLF, Flüelastrasse 11, \\ CH-7260 Davos Dorf, Switzerland \\ E-mail: sovilla@slf.ch \\ ${ }^{2}$ Department of Snow and Avalanches, BFW Institute for Natural Hazards and Alpine Timberline, Hofburg-Rennweg 1, \\ A-6020 Innsbruck, Austria \\ ${ }^{3}$ Warning and Prevention Research Unit, WSL Institute for Snow and Avalanche Research SLF, Flüelastrasse 11, \\ CH-7260 Davos Dorf, Switzerland
}

\begin{abstract}
We report impact pressures exerted by three wet-snow avalanches on a pylon equipped with piezoelectric load cells. These pressures were considerably higher than those predicted by conventional avalanche engineering guidelines. The time-averaged pressure linearly increased with the immersion depth of the load cells and it was about eight times larger than the hydrostatic snow pressure. At the same immersion depth, the pressures were very similar for all three avalanches and no dependency between avalanche velocity and pressure was apparent. The pressure time series were characterized by large fluctuations. For all immersion depths and for all avalanches, the standard deviations of the fluctuations were, on average, about $20 \%$ of the mean value. We compare our observations with results of slow-drag granular experiments, where similar behavior has been explained by formation and destruction of chain structures due to jamming of granular material around the pylon, and we propose the same mechanism as a possible microscale interpretation of our observations.
\end{abstract}

\section{INTRODUCTION}

In avalanche engineering, the pressure, $p$, exerted on an obstacle by an extreme avalanche is assumed to be $p=c_{\mathrm{f}} \rho U^{2}$, i.e. $p$ is proportional to the square of the impact velocity, $U$, and to the avalanche bulk density, $\rho$. Shape and rheology effects are taken into account by a drag coefficient, $C_{\mathrm{f}}$. This formulation is also used by the Swiss Guidelines (Salm and others, 1990) and applies to extreme dry avalanches where impact forces are largely correlated with the avalanche kinetic energy (Sovilla and others, 2008a). In practice, as there is no established alternative, the same formula is also used by practitioners for slow, wet-snow avalanches.

Recent measurements indicate that, for avalanches with low Froude numbers, this formula may heavily underestimate the impact pressures (Norem, 1991; Gauer and others, 2007; Baroudi and Thibert, 2009). However, no satisfactory explanation of these discrepancies in terms of possible underlying processes has been given so far.

Sovilla and others (2008a) suggested that the impact pressure of avalanches in the subcritical flow regime may be governed by other processes than those involved in the supercritical flow regime. They reported a preliminary analysis of pressure and velocity data from the Vallée de la Sionne (canton Valais, Switzerland) test site, which shows that the measured impact pressures did not have a significant velocity dependency for Froude numbers less than 1, i.e. for subcritical flows. Furthermore, they observed that the total drag on the pylon strongly depends on the avalanche flow depth. Finally, Sovilla and others (2008a) observed that the amplitude of pressure fluctuations in wet-dense avalanches increases with flow depth, in contrast to pressure fluctuations in dry-dense avalanches, which are larger close to the avalanche surface and smaller lower down in the avalanche body.

Our measurements of drag forces in slow wet avalanche flow may be consistent with theories for granular systems which predict that drag in slow flow varies linearly with flow depth and is independent of fluid velocity (Wieghardt, 1975; Albert and others, 1999, 2000; Chehata and others, 2003). Thus, the calculation of avalanche slow drag on structures may require a different analytical approach than the avalanche dynamics models currently used.

In this paper, we present additional data of slow drag in avalanche flow and use new analysis methods to improve the preliminary analysis of Sovilla and others (2008a). A comparison of our results with slow granular flow experiments provides further evidence of the granular flow behavior of wet-snow avalanches.

\section{INSTRUMENTATION AND METHODS}

We present data from the full-scale avalanche experimental test site Vallée de la Sionne, which is described in detail by Sovilla and others (2008a). Here we analyze large, wetsnow avalanches that started spontaneously in the release zone at $\sim 2700 \mathrm{~m}$ a.s.l. and followed a track of $\sim 1500 \mathrm{~m}$ length down to a zone with artificial obstacles at $1600 \mathrm{~m}$ a.s.I. (Fig. 1a). Several obstacles equipped with instruments were installed on a large open slope with an average inclination of about $21^{\circ}$ (Sovilla and others, 2008b). At this location, large wet avalanches have a maximum flow depth of $3.5-5.5 \mathrm{~m}$ and flow velocities of $1-10 \mathrm{~m} \mathrm{~s}^{-1}$ (Table 1). As there is no lateral confinement, the avalanches may expand to a width of up to $100 \mathrm{~m}$. They finally run out in the wide open slope below the obstacle zone, or reach the bottom of the valley and stop at the slope on the other side of the valley. One of the obstacles is an oval-shaped, $20 \mathrm{~m}$ high pylon, equipped with pressure and velocity sensors (Fig. 1b).

Piezoelectric load cells are mounted on the pylon at $0.5-5.5 \mathrm{~m}$ above the ground with $1 \mathrm{~m}$ vertical spacing (Schaer and Issler, 2001). During winter 2003/04, sensor diameters were 0.1 and $0.25 \mathrm{~m}$. Since winter 2004/05, only 


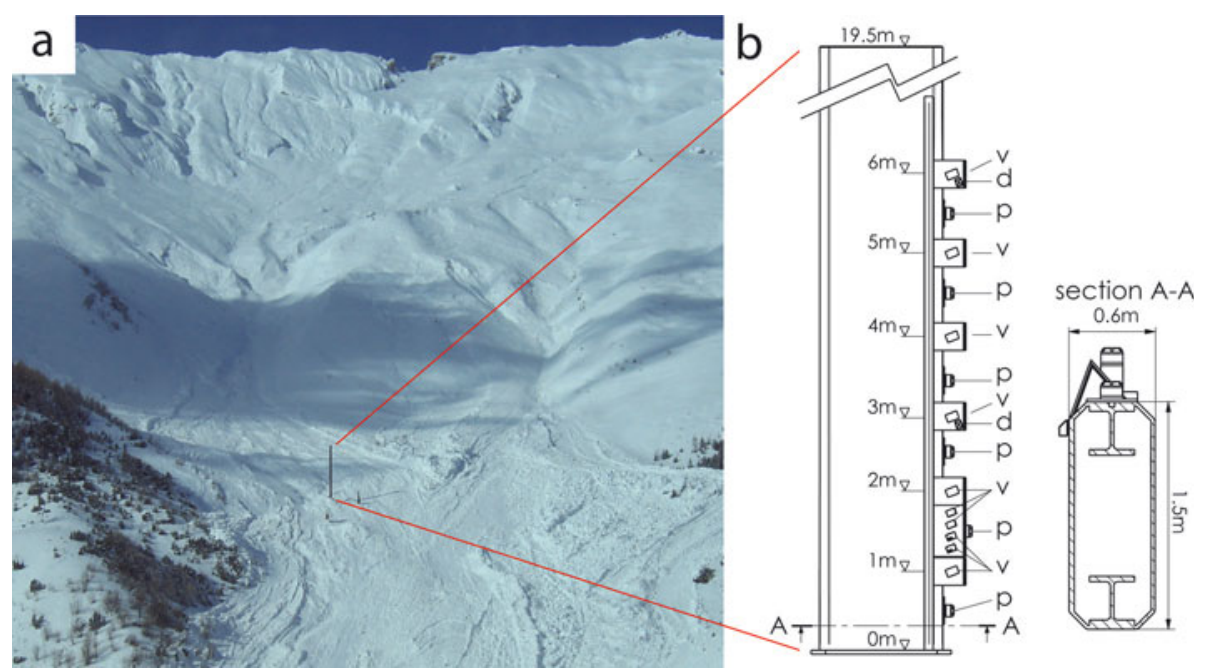

Fig. 1. (a) The avalanche slope at the Vallée de la Sionne test site. A wet avalanche has just hit the obstacle zone. (b) Pylon and sensor installation details ( $p$ is pressure, $v$ is velocity and $d$ is density).

sensors with diameters of $0.10 \mathrm{~m}$ have been used. Pressures are recorded with a sampling frequency of $7.5 \mathrm{kHz}$.

Flow velocities are recorded by optoelectronic sensors mounted flush on wedges, with $1 \mathrm{~m}$ vertical spacing, up to $6 \mathrm{~m}$ above ground. The wedges minimize flow disturbances around the velocity sensors. The velocity is calculated from the cross-correlation between the signals of two reflectivity sensors with $29 \mathrm{~mm}$ streamwise spacing. The sampling frequency is $20 \mathrm{kHz}$. For technical details on the optical velocity measurements and data analysis procedures, see Dent and others (1998) and Kern and others (2009).

We estimated the height of the surface of the flowing avalanche, $h(t)$, at the pylon by using the pressure time series, $p\left(z_{i}, t\right)$, from the load cells at different heights, $z_{i}$, where $i$ indicates the load cells (Fig. 1b). We calculated the centered floating mean, $\left\langle p\left(z_{i}, t\right)\right\rangle$, of the pressure time series using a time-averaging window of $T=1 \mathrm{~s}$. We then defined $h(t)$, the assumed avalanche surface, as the height where the linear interpolation of the two uppermost pressure measurements reached $5 \mathrm{kPa}$.

\section{DATA ANALYSIS}

The three avalanches analyzed had very similar features. Rather than describe all the raw data, we concentrate here on avalanche 8448 (1 March 2007), but we also refer to the other avalanches in our analysis. Table 1 contains some of the avalanche parameters. For a more detailed description of

Table 1. Summary of the wet avalanche events measured at the Vallée de la Sionne test site during the winter seasons 2003/04 and 2006/07

\begin{tabular}{cccccc}
\hline No. & Date & Time & Type & Velocity & $\begin{array}{c}\text { Max. flow } \\
\text { depth } \\
\end{array}$ \\
& & h & & $\mathrm{m} \mathrm{s}^{-1}$ & $\mathrm{~m}$ \\
\hline 6236 & 12 Jan 2004 & 0628 & Natural, wet-dense & $2-7$ & 5.4 \\
6241 & 12 Jan 2004 & 1033 & Natural, wet-dense & $3-8$ & 4.4 \\
8448 & 1 Mar 2007 & 2119 & Natural, wet-dense & $1-3$ & 3.4 \\
\hline
\end{tabular}

the avalanche characteristics see Sovilla and others (2008a) and Kern and others (2009).

A plot of the raw pressure time series, $p\left(z_{i}, t\right)$, of avalanche 8448 is given in Figure 2a. Figure $2 \mathrm{~b}$ shows the time-averaged pressure, $\left\langle p\left(z_{i}, t\right)\right\rangle$, for the same avalanche. Figure $2 c$ gives the corresponding fluctuations, which we characterized by the standard deviation, $\sigma_{p}\left(z_{i}, t\right)$, of the pressure time series:

$$
\sigma_{p}\left(z_{i}, t\right)=\sqrt{\left\langle\left(\left(p\left(z_{i}, t\right)-\left\langle p\left(z_{i}, t\right)\right\rangle\right)^{2}\right\rangle\right.} .
$$

A real avalanche rarely flows in a pure wet- or dry-flow regime. Most of the time, both regimes are present. Typically, in wet avalanches, a plug flow core may be surrounded by dilute flow, particularly if the avalanche was released from altitudes where the snow is still dry, and the snow in the track then becomes wet at lower altitudes.

We consider only measurements belonging to the slowdrag avalanche flow regime and therefore we define below a criterion based on the ratio between fluctuations and average pressure, i.e. on the relative fluctuations. This criterion is based on the fact that pressure fluctuations differ strongly between dry-dense and wet-dense flow (Sovilla and others, 2008a).

Figure $2 \mathrm{~d}$ shows the relative fluctuations of avalanche 8448, $\sigma_{p} /\langle p\rangle$, where $\sigma_{p}$ stands for $\sigma_{p}\left(z_{i}, t\right)$ and $\langle p\rangle$ for $\left\langle p\left(z_{i}, t\right)\right\rangle$.

Figure 3 shows a statistical distribution of the relative fluctuations, $\sigma_{p} /\langle p\rangle$, for each sensor and for each avalanche. On average, $\sigma_{p} /\langle p\rangle$ is 0.21 , with $95 \%$ of points being distributed in the interval $[0.05,0.4]$. This scaling ratio persists for almost all measurements, at all immersion depths and for all avalanches. In Figure $2 \mathrm{~d}$, those points with a scaling ratio lower than 0.05 or higher than 0.4 are marked in grey. In Figure $2 b$, the same points are marked with large dots. Most of the outliers can be related to measurements from the avalanche surface, the avalanche front or the avalanche tail. These points probably originate from a more dilute layer surrounding the avalanche dense core and are excluded from further analysis.

Figure 4a shows pressure measurements that scale with the corresponding fluctuations (according to Fig. 2d) as a 

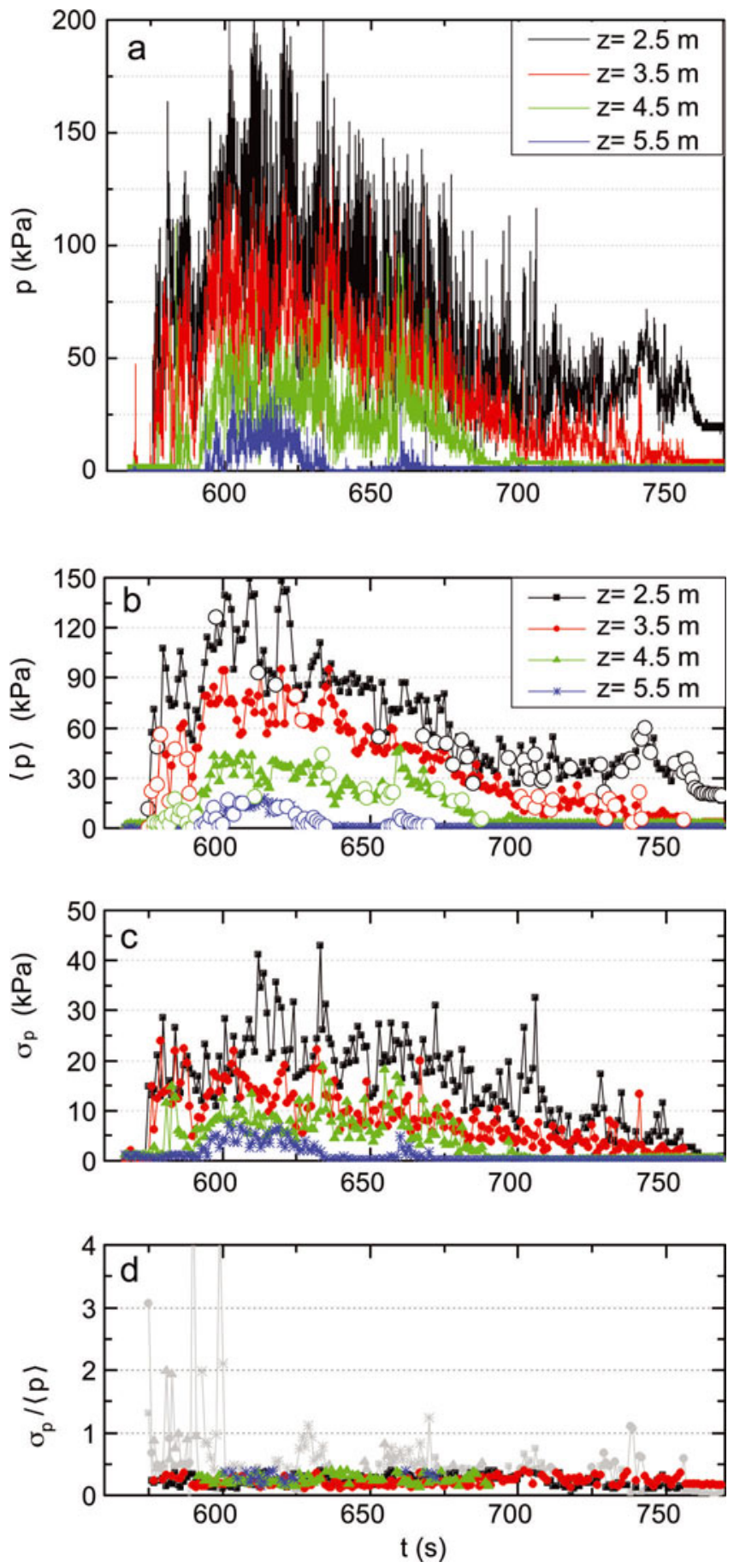

Fig. 2. Avalanche 8448. (a) Raw pressure data; (b) the average pressure, $\langle p\rangle$; (c) fluctuations described by the standard deviation, $\sigma_{p}$; and (d) the scaling function, $\sigma_{p} /\langle p\rangle$. Points not scaling are indicated in gray in (d) and with large dots in (b).

function of flow depth for avalanche 8448 . Figure $4 \mathrm{~b}$ shows a merging of all avalanche pressure profiles.

Figure 5 shows time-averaged velocity profiles measured in the bulk of avalanches 8448, 6236 and 6241 . The profile shapes indicate low-shear or even non-shear flow behavior (plug flow).

\section{DISCUSSION}

Current avalanche engineering design rules cannot predict correctly the forces exerted by wet-snow avalanches. They predict far smaller forces than those we measured (Sovilla

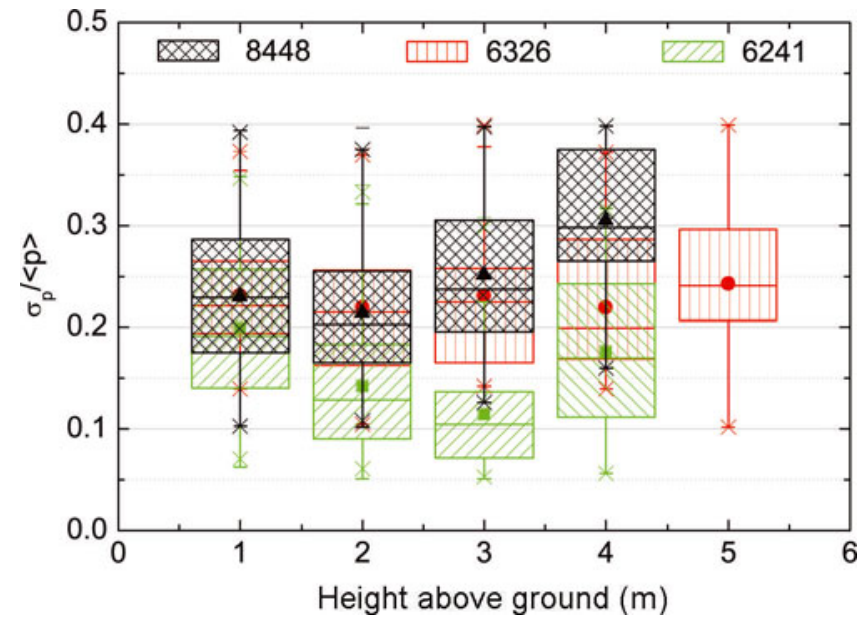

Fig. 3. Statistics of fluctuations and average pressures ratio, $\sigma_{p} /\langle p\rangle$, for each sensor of avalanches 8448, 6231 and 6241. Box plots show mean (symbol in box), median (line in box), $25 / 75 \%$ quantiles (box), 5/95\% quantiles (whiskers) and 0/100\% quantiles (cross).

and others, 2008a) and do not describe the depth dependency we found in the experimental data (Fig. 4a and b). Moreover, the scaling between pressure fluctuations, $\sigma_{p}$, and mean pressure, $\langle p\rangle$, (Fig. $2 \mathrm{~d}$ ) cannot be explained by either fluid-dynamic or particle-impact models.

In fact, our experimental data may be better understood using a slow-drag granular model. Geng and Behringer (2005) reported the scaling between fluctuations and average pressure as typical for slow drag in granular experiments. In these experiments, the drag force experienced by an object moving through a granular medium has been shown to originate from an apparently random distribution of force chains that depart from the object and disperse into the bulk of the granular medium. The drag fluctuations that typically occur in these experiments arise from the formation and rupture of these chains.

The investigation described in this paper, however, clearly differs from slow-drag granular experiments in that the latter typically use granular material with monodisperse elastic disks while, in our case, avalanches are characterized by a polydisperse granular mixture of plastic, compressible and breakable snow clusters.

Furthermore, slow-drag granular experiments are performed in small containers where boundary conditions normally play an important role (Wieghardt, 1975; Albert and others, 1999; Geng and Behringer, 2005), and the force chains that form during these experiments are typically confined in direction and length. In contrast, our experiments are not influenced by lateral confinement, as the pylon on the study site is on an open slope where an avalanche is normally several hundred meters long, up to $100 \mathrm{~m}$ wide and the flow depth can be as high as $5 \mathrm{~m}$.

Nevertheless, none of these differences seems, in principle, to be an obstacle to the formation of force chains. In fact, a polydisperse granular mixture allows for a denser particle packing, and may also favor chain branching and increase the stability of the force chains, even when they are deformed. The absence of lateral confinement may allow the stress chains to develop in any direction, and to cover large areas. Furthermore, small shear rates, and thus small relative displacements between particles, may promote the formation of three-dimensional chains. Our wet avalanches 

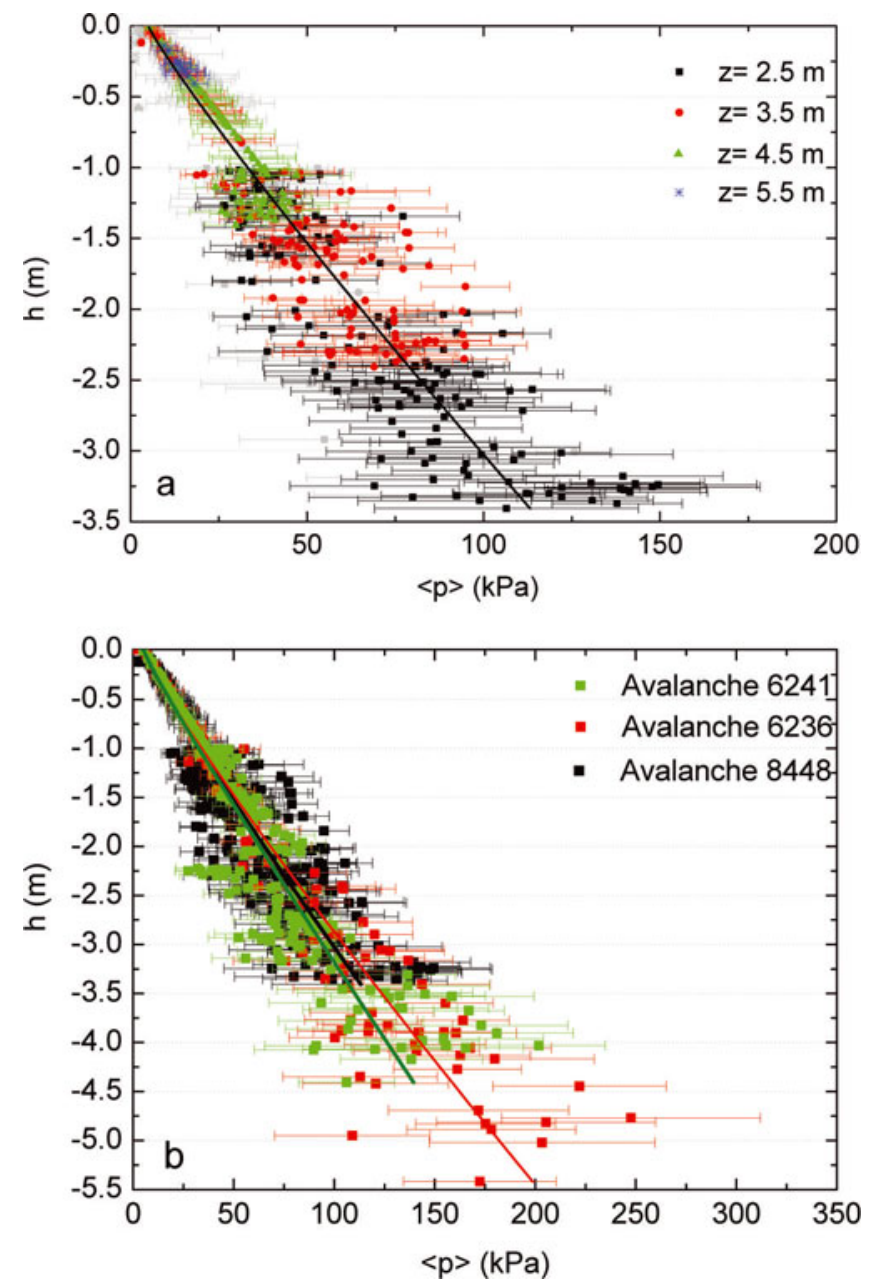

Fig. 4. (a) Mean pressures and fluctuations (pressure standard deviations) as a function of flow depth for avalanche 8448. Gray dots show measurements that were excluded from the analysis because $\sigma_{p} /\langle p\rangle$ did not scale. (b) Pressure and fluctuation standard deviations as a function of flow depth for avalanches 8448, 6236 and 6241. Fitting lines for $p=\zeta \rho g H$ are inserted.

preferably move in a plug-flow regime and are thus characterized by a small shear rate in the core (Sovilla and others, 2008a; Kern and others, 2009). In the bulk of avalanches 8448,6236 and 6241 (Fig. 5), shear rates, $\dot{\gamma}$, are $0.24,0.01$ and $-0.16 \mathrm{~s}^{-1}$. Thus, from a microscopic point of view, fluctuations in our pressure measurements can be interpreted as an indication of the formation and rupture of stress chains due to snow jamming around the pylon.

At the macroscopic scale, slow-drag laboratory experiments with monodisperse granular material showed that the average drag force on a cylinder is $F_{\mathrm{D}}=\eta \rho g d_{\mathrm{C}} H^{2}$, where $H$ is the immersion depth of the cylinder, the dimensionless parameter, $\eta$, characterizes the grain properties, $d_{c}$ is the cylinder diameter, $\rho$ is the flow bulk density and $g$ is the gravitational acceleration (Wieghardt, 1975; Albert and others, 1999). Our data give similar results to those found by Wieghardt (1975) and Albert and others (1999). The straight lines in Figure $4 \mathrm{a}$ and $\mathrm{b}$ show linear fits of the form $p=\zeta \rho g H$, where $\zeta$ is the fitting parameter.

From permittivity measurements performed inside avalanche $8448,3 \mathrm{~m}$ above ground, we estimated the flow density to be $\rho=400 \pm 80 \mathrm{~kg} \mathrm{~m}^{-3}$ (Louge and others, 1997). We used this value to indicate the average density for all

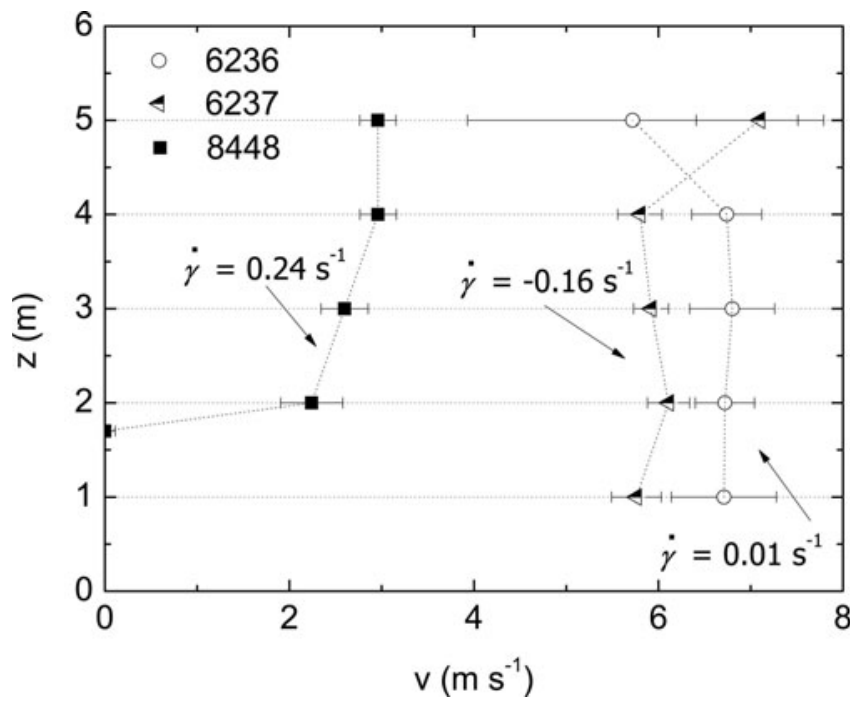

Fig. 5. Velocity profiles and shear rates, $\dot{\gamma}$, extracted from the bulk of avalanches 8448, 6236 and 6241 . Time periods, $620 \mathrm{~s} \leq t \leq 660$ $\mathrm{s}$ for avalanche $8448,530 \mathrm{~s} \leq t \leq 535 \mathrm{~s}$ for avalanche 6236 , and $205 \mathrm{~s} \leq t \leq 215 \mathrm{~s}$ for avalanche 6241 are shown.

avalanches. The best fits resulted for $\zeta=7.2 \pm 2.1,8.1 \pm 1.6$ and 7.6 \pm 1.7 , for avalanches 8448, 6236 and 6241, respectively. The linear relation, $p=\zeta \rho g H$, is compatible with the drag force relation, $F_{\mathrm{D}}=\eta \rho g d_{\mathrm{C}} H^{2}$, found by Wieghardt (1975) and Albert and others (1999). Indeed, Albert and others (1999) observed that in general $F_{\mathrm{D}}$ was linearly proportional to $d_{\mathrm{c}}$. But they also found an important deviation from linearity, when the particle diameter, $d_{g}$, was large compared with $d_{\mathrm{c}}$.

Albert and others (1999) explained the increased drag for low $d_{\mathrm{C}} / d_{\mathrm{g}}$ by the fact that even when $d_{\mathrm{c}} \rightarrow 0$, the cylinder must push grains aside as it moves relative to the medium and must therefore approach a threshold value of $F_{\mathrm{D}}$ for $d_{C} \rightarrow 0$. Albert and others (1999) reported this phenomenon from experiments in the approximative range $0.6<d_{\mathrm{C}} / d_{\mathrm{g}}<3$. In our experiments, assuming $d_{\mathrm{c}}=0.60 \mathrm{~m}$ and $d_{\mathrm{g}}$ varying in the range $0.07-0.12 \mathrm{~m}$ (from granulometry observations in wet avalanche deposits by Bartelt and McArdell (2009)), we obtain $5<d_{\mathrm{C}} / d_{\mathrm{g}}<8$. Therefore, since $d_{\mathrm{C}} / d_{\mathrm{g}}$ for the avalanches we analyzed is in the same order of magnitude as found by Albert and others (1999), we expect that increasing the pylon diameter will result in a lower coefficient $\zeta$.

Another striking characteristic of our experimental data is shown in Figure $4 \mathrm{~b}$ where all avalanche pressure profiles are merged: the average pressures measured at a given immersion depth are in the same range for all avalanches. In the framework of slow-drag granular mechanics, this can be explained by assuming that the three avalanches had a similar granulometry and density. Slow-drag experiments performed by Geng and Behringer (2005) showed that density strongly influences the avalanche drag. For densities above a minimum threshold value, force chains evolve and average pressure increases rapidly with density (Geng and Behringer, 2005). Thus, if the investigated avalanches had a different flow density, this should have led to varying drag. The assumption that the wet avalanches we analyzed had a similar density is also in agreement with recent granulometric investigations of avalanche deposits that show a very similar particle size distribution for a variety of wet and dry 
avalanches. In particular, Bartelt and McArdell (2009) analyzed wet-snow avalanche deposits and observed a log-normal granular size distribution with a median particle size of approximately $0.07-0.12 \mathrm{~m}$. Snow boulders of $>0.5 \mathrm{~m}$ diameter were found on the avalanche surface, and the smallest grain size was in the order of $0.02 \mathrm{~m}$.

Finally, we analyzed the influence of the avalanche velocities, $v$, which for the avalanches we studied (Table 1) were in the range $1-10 \mathrm{~m} \mathrm{~s}^{-1}$. No apparent velocity dependency can be seen in the merged pressure profiles of Figure 4b. Such a dependency would be immediately visible, as it would corrupt the fit of the profiles. Figure 6 shows the dependency of the mean pressure, $\langle p\rangle$, of avalanche 8448 on its velocity. The velocity dependency is very weak and almost non-perceptible. This weak dependency has also been reported in slow-drag granular experiments (Wieghardt, 1975; Albert and others, 1999; Geng and Behringer, 2005).

\section{CONCLUSIONS}

Analyzing pressure signals from wet-snow avalanches, which are characterized by both plug- and dilute-flow regimes, we found that relative fluctuations of the signal are a good criterion for distinguishing between the two regimes. We observed that, for wet-snow avalanches moving in a plug-flow regime, the impact pressure on a pylon increases linearly with flow depth and the pressure is independent of the avalanche velocity. Furthermore, the measured average impact pressure is about eight times larger than the hydrostatic snow pressure. Even if we account for the effects of the small pylon width, average pressures are up to an order of magnitude larger than those predicted by standard engineering rules (Sovilla and others, 2008a).

It is possible that, so far, the drag forces arising from slow avalanche flow have been considerably underestimated since such avalanches have usually been assumed to have fluid-like behavior and to involve viscous and collisional particle interaction as underlying processes (Salm and others, 1990). Thus, our results have direct relevance for estimates of drag forces in design procedures for avalancheprone structures.

A sound theoretical basis for calculating the force-chainrelated pressures on obstacles is missing, but simple fit templates (e.g. Albert and others, 1999) may still help to predict the drag exerted by a slow-moving avalanche on a narrow obstacle better than current avalanche dynamics formulas do. Alternatively, Coulomb's theory for passive failure in a Mohr-Coulomb material may also explain the linear dependency of pressure with flow depth and may be a future solution for the calculation of slow drag in avalanche flow (Wieghardt, 1975). Better predictions should help to prevent incorrect dimensioning of avalanche protection measures which might have fatal consequences.

We also found that the avalanche pressure fluctuations scaled to the average pressure and the average pressures measured at a given immersion depth are in the same range for all avalanches. These features can be explained in the framework of slow drag in granular media. Accordingly, we interpret the pressure fluctuations as indicating the formation and rupture of such force chains.

With our current level of knowledge, we are far from a proof of the existence of force chains in dense avalanche flow and from a sound theoretical description of their

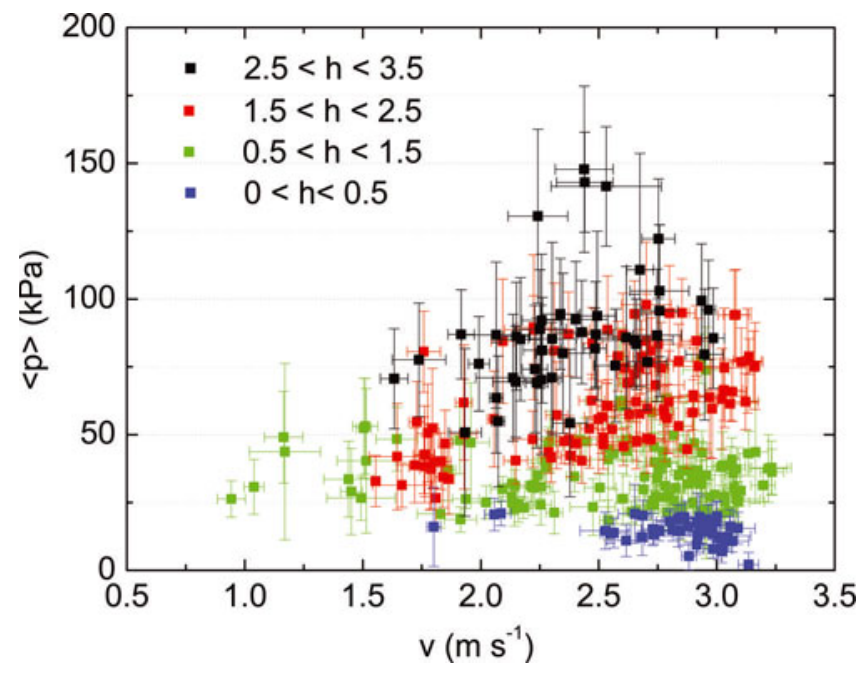

Fig. 6. Pressure as a function of velocity for avalanche 8448 . We indicate measurements from the same range of immersion depth by color.

formation and geometry. Nevertheless, we have been able to provide various indications that the force chains observed in granular experiments may also be present in natural gravity flows of polydisperse inelastic granular mixtures, such as wet-snow avalanches, and that these can also be studied in situations where their flow is not laterally confined.

\section{ACKNOWLEDGEMENTS}

Part of this work was funded by the Swiss National Foundation under grant No. 206021-113069/1. We thank F. Dufour for organizing the field campaigns, and canton Valais, Switzerland, for providing valuable financial support. Special thanks are due to the mechanical and electronic staff of SLF for all their invaluable help.

\section{REFERENCES}

Albert, I. and 8 others. 2000. Jamming and fluctuations in granular drag. Phys. Rev. Lett., 84(22), 5122-5125.

Albert, R., M.A. Pfeifer, A.-L. Barabási and P. Schiffer. 1999. Slow drag in a granular medium. Phys. Rev. Lett., 82(1), 205-208.

Baroudi, D. and E. Thibert. 2009. An instrumented structure to measure avalanche impact pressure: error analysis from Monte Carlo simulations. Cold Reg. Sci. Technol., 59(2-3), 242-250.

Bartelt, P. and B.W. McArdell. 2009. Granulometric investigations of snow avalanches. J. Glaciol., 55(193), 829-833.

Chehata, D., R. Zenit and C.R. Wassgren. 2003. Dense granular flow around an immersed cylinder. Phys. Fluids, 15(6), 1622-1631.

Dent, J.D., K.J. Burrell, D.S. Schmidt, M.Y. Louge, E.E. Adams and T.G. Jazbutis. 1998. Density, velocity and friction measurements in a dry-snow avalanche. Ann. Glaciol., 26, 247-252.

Gauer, P. and 7 others. 2007. On full-scale avalanche measurements at the Ryggfonn test site, Norway. Cold Reg. Sci. Technol., 49(1), 39-53.

Geng, J. and R.P. Behringer. 2005. Slow drag in two-dimensional granular media. Phys. Rev. E, 71(1), 011302. (10.1103/ PhysRevE.71.011302.)

Kern, M., P. Bartelt, B. Sovilla and O. Buser. 2009. Measured shear rates in large dry and wet snow avalanches. J. Glaciol., 55(190), 327-338.

Louge, M.Y., R. Steiner, S.C. Keast, R. Decker, J.D. Dent and M. Schneebeli. 1997. Application of capacitance instrumentation 
to the measurement of density and velocity of flowing snow. Cold Reg. Sci. Technol., 25(1), 47-63.

Norem, H. 1991. Estimating snow avalanche impact pressure on towers. Eidg. Inst. Schnee- und Lawinenforsch. Mitt. 48, 42-56.

Salm, B., A. Burkard and H. Gubler. 1990. Berechnung von Fliesslawinen: eine Anleitung für Praktiker mit Beispielen. Eidg. Inst. Schnee- und Lawinenforsch. Mitt. 47.

Schaer, M. and D. Issler. 2001. Particle densities, velocities and size distributions in large avalanches from impact-sensor measurements. Ann. Glaciol., 32, 321-327.
Sovilla, B., M. Schaer, M. Kern and P. Bartelt. 2008a. Impact pressures and flow regimes in dense snow avalanches observed at the Vallée de la Sionne test site. J. Geophys. Res., 113(F1), F01010. (10.1029/2006JF000688.)

Sovilla, B., M. Schaer and L. Rammer. 2008b. Measurements and analysis of full-scale avalanche impact pressure at the Vallée de la Sionne test site. Cold Reg. Sci. Technol., 51(2-3), 122-137.

Wieghardt, K. 1975. Experiments in granular flow. Annu. Rev. Fluid Mech., 7, 89-114.

MS received 12 March 2010 and accepted in revised form 5 June 2010 